Cahiers $d u$ MONDE RUSSE

\section{Cahiers du monde russe}

Russie - Empire russe - Union soviétique et États indépendants

$46 / 1-2 \mid 2005$

La Russie vers 1550

\title{
La similitude du dissemblable
}

La Russie et la grande-principauté de Lituanie XIV - -milieu du XVI ${ }^{\mathrm{e}}$ siècle

\section{Elena RUSINA}

\section{(2) OpenEdition}

\section{Journals}

\section{Édition électronique}

URL : https://journals.openedition.org/monderusse/8774

DOI : $10.4000 /$ monderusse. 8774

ISSN : $1777-5388$

\section{Éditeur}

Éditions de l'EHESS

\section{Édition imprimée}

Date de publication : 1 janvier 2005

Pagination : $39-50$

ISBN : 2-7132-2055-6

ISSN : $1252-6576$

\section{Référence électronique}

Elena RUSINA, "La similitude du dissemblable », Cahiers du monde russe [En ligne], 46/1-2 | 2005, mis en ligne le 01 janvier 2007, consulté le 04 septembre 2022. URL : http://journals.openedition.org/ monderusse/8774; DOI : https://doi.org/10.4000/monderusse.8774 


\section{CAIR N}

chercher : repérer : avancer

Cet article est disponible en ligne à l'adresse :

http://www.cairn.info/article.php?ID REVUE=CMR\&ID NUMPUBLIE=CMR 461\&ID ARTICLE=CMR 4610039

\section{La similitude du dissemblable. La Russie et la grande-principauté de Lituanie XIVe-milieu du XVle siècle}

par Elena RUSINA

\section{| Editions de l'EHESS | Cahiers du monde russe}

\section{5/1-2 - Vol 46}

ISSN 1252-6576 | ISBN 2713220556 | pages 39 à 50

Pour citer cet article :

- RUSINA E., La similitude du dissemblable. La Russie et la grande-principauté de Lituanie XIVe-milieu du <marquage typemarq="petitecap">xvie siècle, Cahiers du monde russe 2005/1-2, Vol 46, p. 39-50.

Distribution électronique Cairn pour les Editions de l'EHESS.

(C) Editions de l'EHESS. Tous droits réservés pour tous pays.

La reproduction ou représentation de cet article, notamment par photocopie, n'est autorisée que dans les limites des conditions générales d'utilisation du site ou, le cas échéant, des conditions générales de la licence souscrite par votre établissement. Toute autre reproduction ou représentation, en tout ou partie, sous quelque forme et de quelque manière que ce soit, est interdite sauf accord préalable et écrit de l'éditeur, en dehors des cas prévus par la législation en vigueur en France. Il est précisé que son stockage dans une base de données est également interdit. 


\title{
LA SIMILITUDE DU DISSEMBLABLE
}

\author{
La Russie et la grande-principauté de Lituanie \\ XIV'-milieu du XVI ${ }^{\mathrm{e}}$ siècle
}

Expliquer les dissemblances entre les différentes branches des Slaves orientaux, tant sur le plan de la mentalité, des traditions, que de la culture politique et spirituelle, par une divergence de destin historique, est devenu un poncif. De fait, les territoires occupés de nos jours par les Ukrainiens et les Biélorusses ont fait partie de la grande-principauté de Lituanie jusqu'à l'Union de Lublin en 1569, tandis que le noyau principal des territoires russes passa vers la fin du Xve siècle sous le contrôle des grands-princes moscovites.

Malgré le caractère quelque peu simpliste de ce schéma (notons ainsi que certaines villes russes, telles Brjansk ou Smolensk, ont pendant longtemps fait partie de la Lituanie), il est fermement ancré dans l'historiographie actuelle. On peut s'étonner, dans ces conditions, que les chercheurs ne se soient guère posé une question pourtant toute naturelle : comment, sur les débris de l'ancienne Rus', ont pu se constituer deux entités politiques si différentes, obéissant à des logiques historiques opposées, et cela presque simultanément, puisque la Lituanie, tout comme la Moscovie, apparaît sur l'arène politique au XIII siècle ?

Si l'évolution de l'État moscovite le conduisait imperturbablement vers une monarchie centralisée, la grande-principauté de Lituanie se développait, elle, sous le signe du maintien et du renforcement des tendances régionalistes. Voici déjà près d'un siècle, N. A. Maksimejko, spécialiste des assemblées (sejm) lituaniennes, notait que l'orientation vers le particularisme local conduisait à la création d'un État fondé sur le principe de la fédération territoriale. Ceci expliquerait le retour, au XVI siècle, de la grande-principauté de Lituanie à la situation existant au XIVe siècle, au début de l'expansion lituanienne, c'est-à-dire à une mosaïque de territoires autonomes' ${ }^{1}$.

1. N. Maksimejko, Sejmy Litovsko-Russkogo gosudarstva do Ljublinskoj unii 1569 g. [Les Diètes de l'État russo-lituanien jusqu'à l'Union de Lublin de 1569], Kharkov, 1902, p. 38-43. 
Peut-on même parler de tendances centralisatrices au sein de l'État lituanien, comme le croyait Maksimejko ? Actuellement, la majeure partie des chercheurs semble adhérer au stéréotype, devenu article de foi, selon lequel le grand-prince lituanien Vitovt (Vytautas) (1392-1430), contemporain et beau-père du prince Basile Ier de Moscou, aurait été le partisan et le précurseur de la centralisation à venir. Pourtant, une étude plus approfondie de la question, ainsi que (facteur tout aussi important) la découverte de documents du XIVe siècle dans des copies plus tardives ${ }^{2}$, remettent en question la vision d'un Vitovt cherchant délibérément à liquider les apanages. F. I. Leontovič a ainsi remarqué fort justement que ces derniers ne furent pas entièrement supprimés par Vitovt, et que les princes apanagés n'ont pas tous été dégradés au rang de princes au service du souverain ${ }^{3}$.

Le sort de la principauté de Kiev est particulièrement parlant. Elle est communément considérée comme une des « victimes » de la politique centralisatrice de Vitovt, qui priva le prince local Vladimir Olgerdovič (fils d'Algirdas) de son apanage. Les historiens ont tendance à oublier que cette destitution n'a en rien abaissé le statut de la principauté, puisque son nouveau maître fut Skirgajlo (Skirgailla), le compagnon le plus proche du roi Jagellon, et de facto co-souverain de la Lituanie. À la mort de Skirgajlo, ce fut au tour des princes Gol'šanskij - Ivan Ol'gimontovič (Olgimuntowicz) et ses deux fils André et Michel, fondateurs de la première dynastie kiévienne d'origine lituanienne - de monter sur le trône de Kiev pour plus de trente ans. Cette lignée sera remplacée dans les années 1440 par des descendants de Vladimir Ol'gerdovič4 .

Au fond, le résultat principal du règne de Vitovt fut davantage le renforcement de son pouvoir personnel que la centralisation de l'État lituanien. Étant donné le culte médiéval de la puissance, c'est cela qui suscita l'admiration des contemporains, qui ont décrit son règne en termes panégyriques ${ }^{5}$, et de la postérité - jusques et y compris Ivan le Terrible. Cependant, il ne faut jamais oublier avec quelle force se sont manifestées les tendances centrifuges dans la principauté juste après la mort de Vitovt, lors de l'arrivée de Svidrigajlo au pouvoir.

Il est intéressant de voir comment Ivan le Terrible rapprochait l'opposition entre Svidrigajlo et son frère Jagellon de son propre conflit avec le prince Vladimir Andreevič de Starica ${ }^{6}$. Encore plus symptomatique, la réaction du grand-père

2. Cf. l'article particulièrement intéressant à cet égard de J. Tęgowski, « Sprawa przyłączenia Podola do Korony Polskiej w końcu XIV wieku », Teki Krakowskie, 5, 1997, p. 155-176.

3. F. Leontovič, Soslovnyj tip territorial'no-administrativnogo sostava Litovskogo gosudarstva $i$ ego pričiny [La nature sociale de la composition territoriale et administrative de l'État lituanien et ses racines], Saint-Pétersbourg, 1895, p. 30.

4. O. Rusyna, "On the Kyivan Princely Tradition from the Thirteenth to the Fifteenth Centuries", Harvard Ukrainian Studies, 18 (3/4), 1994, p. 180-182.

5. On peut citer, parmi les exemples classiques, l'Éloge de Vitovt ainsi que le poème de Nicolas Hussowski [Hussovianus], Carmen de statura, feritate ac venatione bisontis [Poème traitant de la taille, des mœurs sauvages et de la chasse de l'auroch], composé à Rome en 1521-1522. Beaucoup moins connues, les additions au Livre des Rois du sous-diacre Mikula (NovgorodSeverskij, 1428). Voir O. Rusyna, Ukraïna pid tatarami i Litvoju [L'Ukraine sous les Tatars et la Lituanie], Kiev, 1998, p. 105.

6. Voir R. Skrynnikov, Ivan Groznyj [Ivan le Terrible], Moscou, 1975, p. 89. 
d'Ivan le Terrible, Ivan III, aux problèmes intérieurs de la Lituanie de son temps. En 1496, apprenant qu'il était question d'accorder un apanage à Sigismond, frère du grand-prince de Lituanie Alexandre, resté à l'écart du pouvoir après la disparition de leur père Casimir Jagellon, Ivan III presse sa fille Hélène, l'épouse d'Alexandre, de rappeler à son époux «à quel désordre fut en proie la Lituanie, lorsqu'il y avait pléthore de souverains ». Ivan III compare implicitement l'état de la Lituanie à celui de la Russie sous son père, Basile II 7 .

Ces paroles préfigurent l'incompatibilité future entre les deux modèles étatiques, incompatibilité qui entraîna plus tard la faillite des projets d'unification de la Russie avec la Rzeczpospolita (la «République » polonaise). Les événements sanglants du Temps des Troubles en furent le résultat, événements que le bojarin Théodore Šeremetev, ex-partisan du prince Wladislaw, s'adressant aux commissaires polonais, résumait en ces termes : «Nous ne voulons ni de vos libertés, ni de vos franchises ${ }^{8}$.

Le problème est de déterminer le moment où s'amorce la divergence entre les deux systèmes socio-politiques. Il est certain que ce ne fut pas la diète de Lublin, lorsque la Lituanie fut pratiquement engloutie par la Pologne. Déjà Ivan III percevait une différence foncière entre les États russe et lituanien. Il est intéressant de noter que si les transfuges lituaniens du XIVe et du début du XV siècle se sentaient relativement à l'aise en Moscovie (où s'installèrent plusieurs maisons issues des nobles qui accompagnaient Svidrigajlo en 1408, incorporées par la suite dans les rangs de la vieille aristocratie moscovite - les princes de Zvenigorod et les descendants de Patrice Narymuntovič, tels les Hovanskij, les Golicyn, les Patrikeev, les Kurakin, etc. ${ }^{9}$ ), l'exil de leurs homologues au début du XVI ${ }^{\mathrm{e}}$ siècle s'avéra bien plus dramatique. Il suffit de rappeler les destins de Michel Glinskij et de Basile Šemjačič, qui ne sont jamais parvenus à s'intégrer dans le modèle socio-politique moscovite ${ }^{10}$.

Après eux, les départs (vyhody) aristocratiques de Lituanie vers la Russie cessèrent entièrement (à une seule exception près, l'engagement du prince Dimitri

7. «Pamjatniki diplomatičeskih snošenij Moskovskogo gosudarstva s Pol’sko-Litovskim gosudarstvom. 1487-1533» [«Documents ayant trait aux relations diplomatiques entre la Moscovie et l'État polono-lituanien. 1487-1533 »], SbRIO, (Saint-Pétersbourg), vol. 35, n 43, 1892 , p. 224-225.

8. J. U. Niemcewicz, Dzieje panowania Zygmunta III [Histoire du règne de Sigismond III], vol. 3, Cracovie, 1860 , p. 97.

9. A. Zimin, Formirovanie bojarskoj aristokratii v Rossii vo vtoroj polovine $X V-$ pervoj treti $X V I V$. [La formation de l'aristocratie en Russie, seconde moitié du XVe-premier tiers du $X V r^{e}$ siècle], Moscou, 1988, p. 29-35, 56-58. Notons, au passage, l'attribution erronée à Patrice Narimuntovič du titre de prince de Zvenigorod, que l'on rencontre encore occasionnellement dans 1'historiographie actuelle (voir, par exemple, O. Horuženko, « Gerby potomstva Patrikija Narimuntoviča, knjazja Zvenigorodskogo » [«Les armoiries des descendants de la maison de Patrice Narimuntovič, prince de Zvenigorod »], Russkij rodoslovec, 1, 2001, p. 25-36), même si cette question est parfaitement élucidée dans les études spécialisées, voir M. Byčkova, Sostav klassa feodalov Rossii $v$ XV $v$ : istoriko-genealogičeskoe issledovanie [La composition de la classe féodale en Russie au XVIe siècle : étude historique et généalogique], Moscou, 1986, p. 33 41 .

10. O. Rusyna, « Šem’jačiči ta Možais'ki », in Istorija Ukraïni v osobah : Litovs 'ko-pol's 'ka doba [«Les Šem'jačiči et les Možajskie », in Histoire de l'Ukraine en portraits : l'époque lituano-polonaise], Kiev, 1997, p. 57-62 ; M. Krom, « Mihajlo Glin’skij », in ibid., p. 62-68. 
Wiśnowicki au service d'Ivan IV, ce qui ne fut qu'un bref épisode dans la biographie agitée du prince ${ }^{11}$ ). Le diplomate papal Jacopo Piso, dans son épître de 1514, va dans le même sens : un des obstacles au départ des orthodoxes de Pologne-Lituanie serait la « tyrannie moscovite $»^{12}$. Ce changement d'attitude au sein de la population orthodoxe est d'autant plus symptomatique qu'en 1480 encore, le roi Casimir mettait en garde les Lituaniens contre les sentiments pro-moscovites des « Ruthènes »" ${ }^{13}$, sentiments que les guerres lituano-moscovites de la fin du Xve siècle mettent en évidence. L'échec des campagnes militaires du même type entreprises par Moscou au cours du premier tiers du XVIe siècle - même dans les cas où la Moscovie jouait la carte de la défense de l'orthodoxie en Lituanie - témoigne sans aucun doute des changements d'attitude survenus parmi les Slaves orientaux de la grande-principauté. Les recherches récentes démontrent que c'était précisément la position adoptée par ces populations qui déterminait le caractère et les résultats des actions militaires entre la Moscovie et la Lituanie ${ }^{14}$. Les études actuelles ne nient pas non plus le fait que «la population russe occidentale éprouvait certaines craintes à l'égard du pouvoir arbitraire et illimité du souverain moscovite $»^{15}$.

Il ne faut pas oublier cependant que les sujets mêmes d'Ivan III n'approuvaient guère les tendances autocratiques qui se sont fait sentir en Moscovie sous son règne : elles n'étaient pas naturelles, ni conformes à la logique profonde des institutions. On cite souvent les paroles de Beklemišev-l'Églantier (Bersen), qui voyait là une violation de la tradition, provoquée par l'arrivée à la cour de la suite grecque de Zoé Paléologue, et mettait les Russes en garde : « Un pays qui change ses coutumes ne dure pas bien longtemps $»^{16}$.

Il existe aussi un autre point de vue, qui soutient que le nouvel ordre moscovite s'est formé sous l'influence directe de la Horde d'Or et des khanats qui lui ont succédé. Ce courant de pensée débouche sur un dilemme, formulé il y a un demi-

11. On connaît bien le jugement extrêmement défavorable porté sur le prince par Ivan le Terrible; à toute question concernant Wiśnowicki, l'ambassadeur moscovite envoyé en Lituanie devait répondre : «Il est venu chez notre souverain, tel un chien, et tel un chien, il est reparti ». Mais personne n'a prêté attention au don que fit le tsar, après la mort tragique du prince et pour le repos de son âme, d'un village et de six hameaux du canton de Domagošči dans le district de Belev; voir V.S. Arsen’ev, «Vkladnaja kniga Brjanskogo Svenskogo monastyrja » [Livre des dons du monastère Svenskij de Brjansk], Izvestija Imperatorskogo Russkogo genealogičeskogo obščestva, (Saint-Pétersbourg), 4, section III, 1911, p. 392.

12. A. Kappeler, Ivan Groznyj im Spiegel der ausländischen Druckschriften seiner Zeit, Berne, 1972 , p. 23, 253.

13. H. Lovmjan'skij, « Russko-litovskie otnošenija v XIV-XV vv. » [« Relations russo-lituaniennes aux XIV ${ }^{\mathrm{e}}-\mathrm{XV} \mathrm{V}^{\mathrm{e}}$ siècles), in Iz istorii russkoj kul'tury [Essais sur l'histoire de la culture russe], tome II, vol. 1, Moscou, 2002, p. 395.

14. M. Krom, Mež Rus ju i Litvoj : zapadno-russkie zemli v sisteme russko-litovskih otnošenij konca $X V$ - pervoj treti XVI V. [Entre Russie et Lituanie : les pays russes occidentaux dans le système des relations russo-lituaniennes, fin $d u X V^{e}$-premier tiers $d u X V I^{\mathrm{e}}$ siècle], Moscou, 1995 , p. 229.

15. Ibid., p. 230.

16. Voir H. Grala, « Człowiek wobec władzy na Rusi Moskiewskiej (XIV-XVI w.) » in Człowek w społeczeństwie średniowiecznym [ « L'individu devant le pouvoir en Russie moscovite, XIVe XVIe siècle », in L'homme dans la société médiévale], Varsovie, 1997, p. 420. 
siècle par Michael Cherniavsky : khan ou basileus ${ }^{17}$ ? Quel facteur extérieur avait bien pu altérer l'évolution naturelle de la Grande Russie ? Il faut dire qu'à ce jour, la balance penche plutôt en faveur des influences de la Horde (surtout chez les auteurs anglophones) $)^{18}$.

On perd de vue, ce faisant, la présence bien tangible du facteur tatar dans l'histoire de la Russie du Sud-Ouest. Ce fut d'abord la soumission directe à la Horde, puis le condominium tataro-lituanien, enfin le voisinage dangereux du khanat de Crimée. C'est à ce facteur qu'il faut attribuer l'existence de modes de vie spécifiquement frontaliers, entre autres le phénomène des cosaques ukrainiens. Cette évolution des territoires proto-ukrainiens et proto-biélorusses a été quelque peu modifiée par l'arrivée des Lituaniens, mais ceux-ci, comme on sait, n'ont pas aboli d'un seul coup les institutions politiques et sociales préexistantes.

Il n'est pas question, bien sûr, de prendre pour parole d'Évangile cette sentence des gouvernants lituaniens, que l'on trouve dans toutes les anthologies : « Nous ne touchons pas à la tradition ni n'introduisons de nouveautés». On a pu prouver qu'elle fut surtout employée dans les documents de la fin du XV et du début du $\mathrm{XVI}^{\mathrm{e}}$ siècle, au moment où la grande-principauté connaissait une vague de changements dynamiques affectant différentes sphères de la vie sociale ${ }^{19}$. Par ailleurs, il est temps de renoncer à l'explication communément admise de la progression des Lituaniens en Russie du Sud-Ouest, progression qui ne doit rien à l'antagonisme lituano-tatar. L'incorporation de ces pays à la grande-principauté fut opérée en vertu d'accords qui maintenaient les territoires occupés par la Lituanie dans leur statut de tributaires de la Horde.

De cette dépendance témoignent les yarlyks de Tokhtamysh (1393), où il exige que Jagellon « après avoir collecté le tribut des pays qui nous sont sujets, le remette à nos ambassadeurs pour versement au trésor» (rédaction ouïghoure [uyghur]) ; «ce que les terres princières [incluses] dans tes territoires donnaient en tribut à la Horde Blanche, est à nous donner comme le nôtre ». C'est uniquement la reconnaissance de la suprématie tatare qui permit à Algirdas d'étendre son contrôle à la Russie du Sud-Ouest, malgré la pression exercée par les Chevaliers teutoniques, et cela, d'un seul coup, et non pas graduellement (comme ce fut le cas du pays de

17. M. Cherniavsky, « Khan or Basileus : An Aspect of Russian Medieval Political Theory », Journal of the History of Ideas, 4, 1959, p. 459-476.

18. Voir J. Pelenski, « State and Society in Muscovite Russia and the Mongol-Turkic System in the Sixteenth Century », in The Contest for the Legacy of Kievan Rus', New-York, 1998, p. 228-243. L'auteur considère les institutions turques comme le prototype de leurs homologues russes, non comme leurs parallèles : les assemblées d'états (zemskie sobory) remonteraient ainsi aux khurultay, le système des bénéfices (ou domaines en précaire, pomest’ja), au soyūrghăl de Kazan, la noblesse aux tarxan, etc. Ce point de vue est partagé par D. Ostrowski, Muscovy and the Mongols: Cross-cultural Influences on the Steppe Frontier, 1304-1589, Cambridge, MA, 1998, p. 21,61.

19. M. Krom, « Starina kak kategorija srednevekovogo mentaliteta (po materialam Velikogo Knjažestva Litovskogo XIV-načala XVII v.) » [« La tradition, catégorie de la mentalité médiévale (à partir de documents de la grande-principauté de Lituanie, XIVe-début du XVII ${ }^{\mathrm{e}}$ siècle) »], in Mediævalia Ucrainica : mental'nist' ta istorija idej [mentalités et histoire des idées], vol. 3 , Kiev, 1994,p. 71-72. 
Smolensk). Ainsi s'explique, soit dit en passant, l'absence de renseignements authentiques concernant le rattachement du gros des pays ukrainiens à l'État lituanien, « l'invisibilité » de l'annexion pour les chroniqueurs contemporain $\mathrm{s}^{20}$.

L'abondance des maisons nobles de souche tatare dans la Russie méridionale des $\mathrm{XV}^{\mathrm{e}-\mathrm{XVI}}$ siècles, le maintien au sein de la structure territoriale de la grande-principauté de Lituanie de fiefs tatars remontant à une époque antérieure (par exemple, la « T'ma de Jagoldaj ») ${ }^{21}$ témoignent d'une politique lituanienne volontairement assimilatrice. Pour ce qui est de l'état de confrontation régissant les rapports lituano-tatars de la seconde moitié du XIVe siècle, il faut préciser qu'il s'agit d'un emprunt de l'historiographie actuelle aux chroniques biélorusses et lituaniennes du XvI ${ }^{\mathrm{e}}$ siècle, dans lesquelles le droit de conquête fut sciemment opposé en guise d'argument juridique aux prétentions dynastiques des souverains moscovites à régner sur l'ensemble des « pays russes ».

D'ailleurs, le motif récurrent de la lutte contre les Tatars apparaît déjà dans la première rédaction des chroniques de la grande-principauté (milieu du Xve siècle). On y trouve notamment le Récit du pays de Podol (qui était alors devenu une pomme de discorde entre la Lituanie et la Pologne), où l'on peut lire que les Lituaniens se sont implantés dans cette région grâce à la défaite subie par les princes tatars locaux (« souverains héréditaires [otčiči i dediči] du pays de Podol »). Comme on peut voir, le but politique du Récit est aussi d'affirmer la légitimité des prétentions des souverains lituaniens sur les territoires de l'Ukraine. À noter que, selon le Récit, les princes lituaniens de la maison des Koryatowicz, « arrivés dans le pays de Podol, refusèrent de verser plus longtemps le tribut aux basqaqs », ce qui est démenti par la charte du «prince et souverain du pays de Podol », Alexandre Koryatowicz (1375), laquelle confirme le maintien de la pratique traditionnelle $\mathrm{du} \ll$ tribut aux Tatars $»^{22}$.

On voit que l'importance du facteur tatar dans l'histoire de la grande-principauté de Lituanie était beaucoup plus prononcée que l'historiographie moderne ne veut bien l'admettre. Le nombre de stéréotypes de ce genre, qui pèsent sur la recherche actuelle, s'explique en grande partie par l'état de décadence prolongée que connurent les études lituaniennes après leur essor fulgurant de la fin du XIXe et du début du XXe siècle. Ce n'est un secret pour personne que l'historiographie soviétique professait une vision mosco-centriste de l'évolution historique des peuples de l'ex-URSS. Toute déviation de ce schéma théorique était censée mener à une impasse. Il est évident qu'une telle approche ne stimulait guère l'analyse objective des processus qui avaient eu pour cadre la grande-principauté de Lituanie, État multiethnique et multiconfessionnel, étranger à toute forme d'intolérance religieuse et d'orthodoxie, doté en outre d'un système d'autonomie régionale et de garanties juridiques s'étendant aux personnes et aux différents corps sociaux.

20. O. Rusyna, Sivers `ka zemlja y skladi Velikogo knjazivstva Litovs 'kogo [La Sévérie au sein de la grande-principauté de Lituanie], Kiev, 1998, p. 79-81.

21. O. Rusyna, «K istorii Jagoldaevoj t'my» [ "Contribution à l'histoire de la "T'ma de Jagoldaj” »), in States, Societies, Cultures : East and West, New York, 2004, p. 1013-1024.

22. « Letopisi belorussko-litovskie » [« Chroniques biélorusses et lituaniennes »], in PSRL, vol. 35, Moscou, 1980, p. 66, 74, 138 ; V. Rozov, Ukraïns 'ki gramoti [Chartes ukrainiennes], vol. 1 , Kiev, 1928, n 10 , p. 20. 
Malgré l'attrait exercé par ce modèle social et étatique, Vilnius était perçue comme fondamentalement inférieure à Moscou en tant que centre d'unification des pays slaves orientaux (dans le cas contraire, selon Vladimir Pašuto, « nous nous verrions contraints d'identifier la politique d'Algirdas avec celle de Dimitri Donskoj... Or, nos sources s'opposent à une telle interprétation » ${ }^{23}$ ).

Il n'y a donc rien d'étonnant à ce que le pionnier de l'étude comparée des deux modèles socio-politiques élaborés par les Slaves orientaux ait été un chercheur américain. Dans son ouvrage, qui met en regard les États russe et polono-lituanien auX XVe-XVI siècles (les statuts respectifs du pouvoir monarchique et de la noblesse sont choisis comme critères principaux de comparaison), Jaroslaw Pelenski insiste, à juste titre, sur l'idéalisation excessive de la centralisation moscovite, devenue un lieu commun de l'historiographie soviétique depuis le milieu des années 1950 :

This particular system was looked upon as the ideal and most progressive among the existing socio-political systems. Muscovite Russia was regarded as having come very close to the ideal model, even when compared with Western monarchical states. Poland allegedly had failed the test of attaining the ideal model because she did not follow the generally applicable historical laws which provided for a transition toward a centralized and absolutistic monarchy. She was assumed to have deviated from the prevailing European tendency and to have developed a very specific and unique system of nobility democracy, which lasted from 1454 to the end of the sixteenth century. This system led to the eventual decay of the Polish-Lituanian Commonwealth and its ultimate downfall ${ }^{24}$.

Paradoxalement, les idées évoquées ci-dessus renvoient, à certains égards, aux idéologèmes moscovites du XVI ${ }^{\mathrm{e}}$ siècle, également examinés par Pelenski. (Il suffit de citer l'épître d'Ivan le Terrible - écrite sous le nom du prince Mihail Vorotynskij - au roi de Pologne et grand-prince de Lituanie, Sigismond-Auguste, où on peut lire : «l'autocratie impériale de nos grands souverains [qui ne tiennent leur pouvoir de personne] ne se compare point à votre royauté misérable $»^{25}$ ).

Malheureusement, le travail de Pelenski passa inaperçu dans l'historiographie soviétique et post-soviétique. Cependant, cette dernière semble adopter une approche plus pondérée à l'égard du problème en question. Des tentatives de comparaison de l'évolution sociale et politique des deux États ont vu le jour. La première fut celle de Margarita Byčkova, qui, dans son ouvrage ${ }^{26}$, examine trois

23. V. Pašuto, B. Florja, A. Horoškevič, Drevnerusskoe nasledie i istoričeskie sud’by vostočnogo slavjanstva [L'héritage de l'ancienne Rus' et les destins historiques des Slaves orientaux], Moscou, 1982, p. 28

24. J. Pelenski, "Muscovite Russia and Poland-Lituania, 1450-1600: State and Society Some Comparisons in Socio-Political Developments", in State and Society in Europe from the Fifteenth to the Eighteenth Century: Proceedings of the First Conference of Polish and American Historians (May 27-29, 1974), Varsovie, 1985, p. 95-96.

25. Poslanija Ivana Groznogo [Epîtres d'Ivan le Terrible], Moscou-Leningrad, 1951, p. 259-260.

26. M. E. Byčkova, Russkoe gosudarstvo $i$ Velikoe knjažestvo Litovskoe s konca $X V V$. do $1569 \mathrm{~g}$ : Opyt sravnitel'no-istoričeskogo izučenija političeskogo stroja [Russie et grandeprincipauté de Lituanie de la fin du XV siècle jusqu'à 1569. Essai d'étude comparée du système politique], Moscou, 1996. 
aspects du problème : les institutions du pouvoir, la structure sociale des élites dirigeantes, la présentation idéologique du pouvoir.

Le texte de l'historienne moscovite a suscité des appréciations mitigées. Ainsi Mihail Krom, dans la conclusion de son compte rendu :

The idea of comparing the political systems of these two neighbouring East European countries must be welcomed and deserves our whole support. However, this brave attempt to carry out such an ambitious project can hardly be considered successful ${ }^{27}$.

Cependant, malgré tout ce que l'on peut reprocher au travail de l'auteur (pauvreté des sources, méconnaissance de bon nombre de travaux fondamentaux concernant le sujet, affirmations avancées sans preuves, amalgame injustifié des institutions polonaises et lituaniennes, manque de conclusions personnelles - M. Byčkova, de son propre aveu, «ne prétend guère apporter de solutions définitives », mais « vise plutôt à poser le problème»), l'historienne $\mathrm{a}$, sans aucun doute, rempli sa fonction essentielle : stimuler la recherche.

En témoigne la parution récente d'un long article de Mihail $\mathrm{Krom}^{28}$, ainsi que l'étude de Hieronym Grala ${ }^{29}$. Les deux auteurs se réfèrent explicitement au livre de Byčkova. Si la première étude prend pour objet les « grandes phases des relations entre les deux États voisins au cours de plusieurs siècles », la seconde préfère la micro-analyse comparative. Au stade où en est l'élaboration du problème, cette dernière approche paraît très féconde : nous devons au chercheur polonais plusieurs observations pertinentes quant à la composition, au statut social, aux perspectives de carrière et au rôle de « ferment culturel » des secrétaires des bureaux moscovites et des scribes de la Chancellerie princière lituanienne.

Ce n'est pas par hasard que la recherche contemporaine entreprend de comparer les phénomènes culturels des deux États. Il faut mentionner, à ce propos, l'étude de Anatolij Turilov ${ }^{30}$. L'auteur soumet à une analyse critique l'idée selon laquelle ce serait un « substrat slave du Sud », propre à la culture de la Russie occidentale, qui la distinguerait de la culture moscovite.

27. Lituanian Historical Studies, 3, 1998, p.161.

28. M. Krom, « Rossija i Velikoe knjažestvo Litovskoe : dva puti v istorii » [« La Russie et la grande-principauté de Lituanie : deux voies historiques»], Anglijskaja naberežnaja, (SaintPétersbourg), $n^{\circ} 4,2000$, p. 73-100.

29. H. Grala, « Diacy i pisarze: wczesnonowożytny aparat władzy w Państwie Moskiewskim i Wielkim Księstwie Litewskim (XVI - pocz. XVII w.) » [« Secrétaires et scribes : l'appareil du pouvoir dans l'État russe et dans la grande-principauté de Lituanie à l'aube des Temps Modernes (XVI - début XVII siècle)»], in Modernizacja struktur władzy w warunkach opóźnienia [Modernisation des structures de pouvoir dans un contexte de retard historique], Varsovie, 1999, p. 73-91.

30. A. A. Turilov, « Južnoslavjanskie pamjatniki v literature i knižnosti Litovskoj i Moskovskoj Rusi XV-pervoj poloviny XVI v.: paradoksy istorii i geografii kul'turnyh svjazej» [ « Euvres littéraires des Slaves du Sud dans la littérature et dans l'érudition de la Rus' moscovite et de la Rus' lituanienne, $\mathrm{XV}^{\mathrm{e}}$ - première moitié du XVI ${ }^{\mathrm{e}}$ siècle : les paradoxes de l'histoire et de la géographie des influences culturelles »], in Slavjanskij al'manah 2000, (Moscou), 2001, p. 247-285. 
Il démontre que l'on connaissait en Moscovie bien davantage de textes d'origine slave méridionale qu'en Pologne-Lituanie, quoique celle-ci fût plus proche des Balkans. Ce paradoxe s'explique, selon lui, par le caractère des relations littéraires et érudites entre Slaves orientaux et méridionaux au Moyen-Âge :

Elles avaient ceci surtout de particulier qu'elles n'étaient ni la conséquence, ni le corollaire des relations politiques et ecclésiales entre États slaves orthodoxes. Elles passaient, la plupart du temps, par Constantinople et par les centres internationaux de la vie monacale orthodoxe (aux XIVe-XVI ${ }^{e}$ siècles, c'était avant tout le mont Athos). Le plus souvent, ces contacts informels ou semi-officiels s'effectuaient par le biais du bas et moyen clergé (clergé régulier surtout), avec quelquefois la participation de l'épiscopat. Dans une situation de ce genre, le facteur de proximité (ou d'éloignement) géographique de telle ou telle région slave orientale par rapport aux Balkans n'exerce d'influence déterminante ni sur le degré d'intensité de ces relations, ni sur leur fécondité31.

D'autres paradoxes de la vie culturelle de la grande-principauté de Lituanie attendent encore leur explication. Ainsi, le pluralisme culturel propre à la Lituanie ${ }^{32}$ aurait dû stimuler l'évolution de la tradition savante slave orientale. Au lieu de cela, nous constatons une nette décadence de la création intellectuelle: le nombre d'œuvres littéraires originales ayant vu le jour en territoire lituanien ne se compare pas avec celui des textes créés dans les pays de la Grande Russie, pourtant infiniment plus homogènes du point de vue culturel. Le même Anatolij Turilov démontre, en étudiant le répertoire des traductions du latin, du polonais et du tchèque réalisées par des lettrés ukrainiens et biélorusses au $\mathrm{XV}^{\mathrm{e}}$ et au début $\mathrm{du}$ $\mathrm{XVI}^{\mathrm{e}}$ siècle ${ }^{33}$, que parmi les œuvres littéraires étrangères, qui représentaient un potentiel culturel colossal, seul un petit nombre de textes fut retenu. Furent traduits essentiellement des apocryphes et des récits de visions. Selon l'auteur :

la hiérarchie catholique n'aurait certainement pas placé un tel choix de textes dans les priorités de la traduction. Cela prouve à l'évidence l'apathie du clergé catholique polonais et lituanien en matière de propagande pour l'Union des Églises (et l'absence de toute velléité de soumettre les «schismatiques»). En même temps, et pour la même raison, les textes traduits ne témoignent pas non plus de l'intérêt des cercles orthodoxes instruits pour le dogme catholique, car ils ne contiennent au mieux que des miettes d'information touchant le catholicisme proprement $\mathrm{dit}^{34}$.

\section{Ibid., p. 264}

32. Cf. E. Banionis, «Litva v XV v. : oščuščenie mnogoobrazija okružajuščego mira » [« La Lituanie au Xve siècle : perception de la diversité du monde environnant »], in Mediœvalia Ucrainica..., op. cit., vol. 3, p. 127-130.

33. A. A. Turilov, «Perevody s latinskogo i zapadnoslavjanskih jazykov, vypolnennye ukrainsko-belorusskimi knižnikami v XV - načale XVI v. » [ "Traductions du latin et des langues slaves occidentales réalisées par des érudits ukrainiens et biélorusses au XVe et au début du XVI ${ }^{\mathrm{e}}$ siècle »], in Kul'turnye svjazi Rossii i Pol'ši, XI-XX vV. [Liens culturels entre Russie et Pologne, $X I^{e}-X X^{e}$ siècle], Moscou, 1998, p. 56-68.

34. Ibid., p. 63-64. 
L'éloignement culturel réciproque des populations orthodoxes et catholiques se manifeste également dans le domaine des chroniques. On sait que les premières chroniques lituaniennes, rédigées au milieu du $\mathrm{Xv}^{\mathrm{e}}$ siècle, opéraient une synthèse entre un texte autochtone, la « Chronique des Grands-Princes de Lituanie » et les informations communes aux chroniques russes, qui couvraient la période allant du « commencement des pays russes » jusqu'au début du XVe siècle. Au XVI siècle, ce schéma cesse de satisfaire les lettrés de la grande-principauté, qui élaborent des théories sur les origines romaines des Lituaniens, et prennent le contre-pied des écrits moscovites, « dénués de toute trace d'antiquité, n'ayant rien qui incite à la bravoure, car la langue ruthène nous est étrangère, à nous Lituaniens, qui sommes Italiens, engendrés par le sang italique $»^{35}$.

Finalement, la partie d'introduction commune aux chroniques russes fut supprimée, laissant place à un récit légendaire sur les origines de l'histoire de la Lituanie. Selon N. A. Morozova, la Chronique des temps passés (Povest' vremennyh let), formellement réfutée par les lettrés lituaniens, devint « une sorte de modèle structural et sémantique, un schéma tout prêt, que l'auteur de la partie légendaire des chroniques lituaniennes remplit d'un contenu différent ${ }^{36}$. Ainsi, même en prenant leurs distances par rapport à l'héritage de l'ancienne Rus', les lettrés de Vilnius restaient fidèles au code culturel commun aux pays russes ${ }^{37}$.

Cependant, même dans ce cas précis, les réalités socio-politiques se faisaient sentir, comme en témoigne la comparaison de la généalogie légendaire des princes lituaniens avec celle des grands-princes moscovites. Margarita Byčkova montre dans son analyse que

la dépendance vassalique des sujets à l'égard du souverain reposait sur des fondements idéologiques qui n'étaient pas les mêmes en Russie et en Lituanie. Le fait d'avoir été depuis toujours au service du prince de Moscou, l'écart infranchissable entre les origines du prince et celles des bojare (lieux communs

35. Mihalon Litvin, O nravah tatar, litovcev i moskvitjan [Michel le Lituanien, De moribus Tartarorum, Lituanorum et Moschorum (1615)], Moscou, 1994, p. 85-86.

36. N. A. Morozova, «O drevnerusskih istočnikah legendarnoj časti letopisej Velikogo Knjažestva Litovskogo » [« Sur les sources russes de la partie légendaire des chroniques de la grande-principauté de Lituanie »], Kalbotyra, (Vilnius), 43 (2), 1993, p. 52-59. On observe la même approche "prédatrice » lorsqu'il s'agit des portraits contenus dans les chroniques russes : ils ont été utilisés, sans se soucier des anachronismes, pour la rédaction du récit légendaire sur la campagne de Gediminas en Volynie et dans le pays de Kiev (Voir O. Rusyna, « Kiïvs’ka viprava Gedimina (tekstologičnij aspekt problemi) » [« La campagne de Gediminas à Kiev (aspects textologiques du problème) »], Zapiski Naukovogo tovaristva imeni Ševčenka, (Lvov), vol. 231, 1996, p. 147-157.) Il est moins évident que la chronique lituanienne, en relatant l'assassinat de Sigismond Keistutovič (fils de Keistutis), se soit inspirée du récit du meurtre du prince André Bogoljubskij (O. Rusyna, «Vid Kuz'mišči-kijanina do kijanina Skobejka (modeljuvannja smerti v "Hronici Bihovcja") » [« Du kiévien Côme au kiévien Skobejko (modélisation de la mort dans la Chronique de Byhov)»], Socium, (Kiev), 1, 2002, p. 37-53).

37. Au même moment, on assiste en Moscovie à des phénomènes diamétralement opposés ce qui n'est pas pour nous surprendre. La politique extérieure des souverains moscovites, dont la pierre angulaire était le principe patrimonial, autrement dit leur droit historique à l'héritage des Rurikides, s'appuyait sur les réalités de l'époque kiévienne. Les Lituaniens ne pouvaient justifier leurs prétentions que par le droit de conquête, comme il a été montré ci-dessus. 
des légendes généalogiques du XVI ${ }^{e}$ siècle) s'inscrivaient parfaitement dans le système qui régissait les relations du grand-prince moscovite avec ses sujets. [...] En Pologne et en Lituanie, en revanche, où l'élection du souverain le rendait plus dépendant des grands seigneurs et de la noblesse, surtout aux XVI $\mathrm{e}_{-}$ $\mathrm{XVII}^{\mathrm{e}}$ siècles où existaient des structures favorisant l'autonomie de celle-ci, une vision généalogique plus égalitaire, mettant sur un même pied le suzerain et ses vassaux, devenait possible ${ }^{38}$.

Ainsi, jusque dans les documents généalogiques, on peut trouver matière à des recherches comparatistes sur l'évolution socio-politique des deux États.

La problématique esquissée dans le présent article est si vaste qu'elle mérite incontestablement un traitement plus détaillé sous la forme d'études monographiques $^{39}$. D'ores et déjà, cependant, on peut entrevoir toute l'ampleur des perspectives de recherche qu'offrent les problèmes soulevés ci-dessus. Comme j'ai essayé de le montrer, il est impossible de les aborder sans remettre en cause tout un ensemble de stéréotypes ayant trait surtout à la grande-principauté de Lituanie. Il faut espérer que le renouveau d'intérêt que suscite actuellement la comparaison entre ces deux modèles sociaux et politiques parallèles permettra d'en élever substantiellement le niveau.

(traduit du russe par André Filler et André Berelowitch)

Académie nationale des sciences d'Ukraine Institut d'histoire de l'Ukraine

Kiev

erusina@ukr.net

38. M. Byčkova, «Ideja vlasti i poddanstva v genealogičeskoj literature XV-XVI vv.» [«L'idée du pouvoir et de la sujétion dans les textes généalogiques des XVe-XVI siècles »], in Istoričeskaja genealogija, (Ekaterinbourg-Paris), 2, 1993, p. 9. La tentative de tracer une sorte de «ligne de partage des eaux » idéologique entre les généalogies légendaires des princes russes et celle des princes lituaniens, comme on la trouve chez A. I. Filjuškin, paraît moins convaincante ( «Legendarnye rodoslovija velikih knjazej litovskih i velikih knjazej moskovskih : principy postroenija » [«Généalogies légendaires des grands-princes russes et lituaniens : principes de composition »], Russkij rodoslovec, 1, 2001, p. 6-14). S'il est incontestable que «les légendes généalogiques reflètent nombre de traits de mentalité politique et d'éléments idéologiques propres aux deux États », les sources analysées par l'auteur n'autorisent guère ses conclusions. Il s'agissait, selon lui, « des modèles, des paradigmes du développement politique et culturel des pays d'Europe orientale, fondés sur un dilemme : soit le rapprochement avec l'Occident (dont le système de valeurs est justement à l'œuvre dans la version lituanienne), soit la création d'un État autochtone édifié sur la tradition historique (même si elle relève quelquefois du mythe) ».

39. Comme par exemple l'étude de K. Zernack, Polen und Russland: Zwei Wege in der europäischen Geschichte, Francfort - Berlin, 1994. 